\title{
Angular momentum evolution of low-mass pre-main sequence stars via extreme coronal mass ejections
}

\author{
A.N. Aarnio ${ }^{1, \star}$, S.P. Matt ${ }^{2}$, and K.G. Stassun ${ }^{3,4}$ \\ 1 Astronomy Department, University of Michigan, 830 Dennison Building, 500 Church Street, Ann Arbor, MI 48109, \\ USA \\ 2 Laboratoire AIM Paris-Saclay, CEA/Irfu Université Paris-Diderot CNRS/INSU, 91191 Gif-sur-Yvette, France \\ 3 Department of Physics and Astronomy, Vanderbilt University, Nashville, TN 37235, USA \\ 4 Department of Physics, Fisk University, Nashville, TN 37208, USA
}

Received 2012 Aug 21, accepted 2012 Dec 5

Published online 2013 Feb 1

Key words stars: activity - stars: flare - stars: pre-main sequence - stars: mass-loss - stars: rotation

The angular momentum evolution of cool stars during the pre-main sequence phase of stellar evolution remains a major outstanding problem. Multiple processes are likely involved in the transfer of mass and angular momentum within and out of the star+disk system. The role of coronal mass ejections (CMEs), energetic events which shed mass and magnetic flux in the Sun, has yet to be fully explored in the context of pre-main sequence stars. It is well established that young, solar-type stars exhibit X-ray activity levels up to four orders of magnitude higher than the present-day Sun, suggesting that CMEs associated with these extreme X-ray flares could be an important process for expelling mass and angular momentum. We present a novel approach to modeling the CMEs of low-mass pre-main sequence stars that uses a solar-calibrated CME model and observed X-ray flare rates for young stars. We derive mass loss rates via stellar CMEs and calculate their attendant angular momentum losses during the pre-main sequence phase. While we find the mass loss rates to be modest, $\sim 10 \%$ of steady-state stellar wind values from the literature, the angular momentum losses can be substantial, potentially counteracting the effects of initial stellar spin-up due to contraction in tens of Myr.

\section{Introduction}

Decades of multi-wavelength, high cadence, high spatial resolution observations have shown complex magnetically active regions and associated energetic phenomena such as flares and CMEs. Understanding the physics underlying these phenomena is important for understanding the magnetic nature of the Sun generally, the physics of mass loss specifically, and the far-reaching effects of space weather on the denizens of $1 \mathrm{AU}$.

During the pre-main sequence phase of stellar evolution, multiple processes transfer mass, and thus angular momentum, between protostar and circumstellar disk, and even out of the system. The "angular momentum budget" of a premain sequence star has multiple sources and sinks, many of these interdependent (e.g., accretion powered stellar winds; Matt \& Pudritz 2005), the relevance of each term timedependent. In the earliest phases of stellar evolution, accretion from a circumstellar disk acts to spin up the protostar, while jets, winds, and episodic outbursts shed angular momentum. Star-disk interaction mediated via stellar magnetic field plays a more difficult to quantify role, potentially acting to simultaneously spin up and slow the stellar rotation (Matt \& Pudritz 2005; Matt et al. 2010; Orlando et al. 2011).

\footnotetext{
^ Corresponding author: aarnio@umich.edu
}

With the discovery of very large magnetic loops confining X-ray emitting plasma in TTS (Favata et al. 2005; Massi et al. 2008; Skelly et al. 2008), questions of their origins, stability, and the ramifications of mass loss from such extended magnetic lever arms followed (Aarnio et al. 2012a and references therein). In this work, we explore the ramifications of mass loss from extended magnetic lever arms: we aim to make first estimates of mass loss rates and spindown times for pre-main sequence stars via stellar coronal mass ejections.

\section{Solar-stellar analogy}

In Aarnio et al. (2011, hereafter A11), by cross-referencing a decade of solar flare and CME data, we established that a number of properties of flares and CMEs are correlated when the flares and CMEs are associated; that is, when a given flare and CME occur simultaneously and cospatially. We found that flare flux and CME mass are log-linearly related over many dex in flux/mass parameter space, with the highest energy flares occurring with the most massive CMEs.

The results of A11 present the CME/flare relationship in terms of flux; for application to young stars, we convert that to energy and extrapolate the relationship up to the regime 


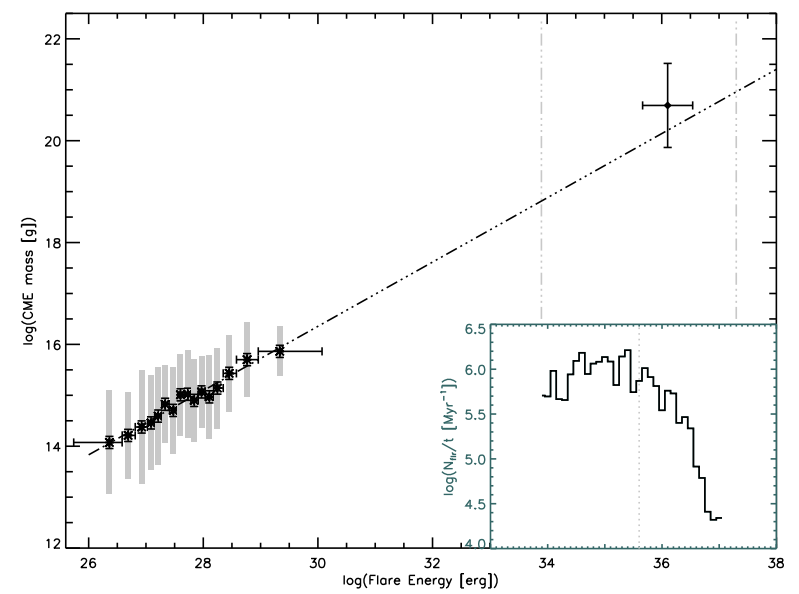

Fig. 1 (online colour at: www.an-journal.org) The solar flare energy/CME mass relationship of A11, plotted with the fit (Eq. (1); dark, triple-dot dashed line) extrapolated up to TTS flare energies (vertical, light, triple-dot dashed lines). The single point represents the mean (error bars are standard deviations) loop mass and flare energy of the 32 Favata et al. (2005) flares. Inset is the X-ray flare energy/rate distribution of Albacete Colombo et al. (2007) for the ONC. Figure directly reproduced from Aarnio, Matt \& Stassun (2012b).

of T Tauri flare energies (see Fig. 1). The functional form of the relationship is

$M=K_{\mathrm{M}} E^{\beta}$,

where $M$ denotes CME mass and $E$ the flare energy. In cgs units, $\beta=0.63$ and $K_{\mathrm{M}}=2.7 \times 10^{-3}$.

At present, we lack data on stellar CMEs (though there have been recent observational attempts with FUSE spectra which yielded ambiguous results; Leitzinger et al. 2011), so we use our solar-calibrated relationship along with X-ray activity rates from recent deep observations of young clusters to estimate mass loss rates on young stars via CMEs. In an effort to place some constraint on the T Tauri energy end of this relationship, we look to one of the closest inferrable masses of coronal structures on young stars: the $\mathrm{X}$-ray emitting plasma in the flaring loops. The X-ray emission measure of the material provides a density, and in the calculation of the loop height, a geometry is assumed (based on spatially resolved solar flare loop observations). Calculating loop masses for the 32 highest energy flares in the Chandra Orion Ultradeep Project (COUP) sample, we find they range from $\sim 10^{19}-10^{22} \mathrm{~g}$; these values scatter about the extrapolated fit (Fig. 1, single point) and likely represent an upper limit to the mass of associated CMEs. Remarkably, these values scatter about the extrapolated solar relationship, 6 dex in energy above the strongest solar flares.

\section{Mass loss rates via extreme CMEs}

To estimate mass lost by thus far unobserved CMEs, we use flares as an observable proxy, inferring a mass distribution

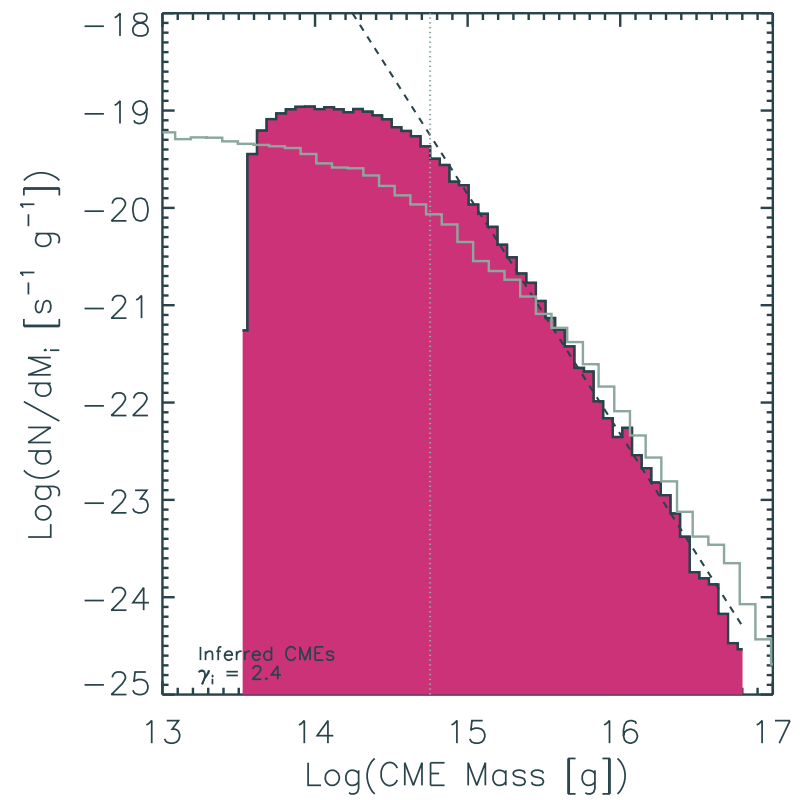

Fig. 2 (online colour at: www.an-journal.org) Solar CME mass distributions: observed (light histogram) and inferred (filled histogram). The dashed line is the analytical fit to the distribution above the flare energy distribution completeness limit (vertical, dotted line). The power law slope, $\gamma_{\mathrm{i}}$, is 2.4 .

for associated CMEs. We first test whether we are able to reproduce the observed solar CME mass loss rate. We perform an empirical calculation first, leaving in the effect of observational bias against the lowest energy flares (which directly translates into a "turning over" of the event rate distributions at low flare energy/CME mass). Then, we attempt to counteract this bias by analytically determining mass loss rates from power law fits to the distributions. We report our results for both the solar and stellar cases.

\subsection{Solar CME mass loss rates}

Summing up the total observed mass lost via CMEs (light distribution, Fig. 2), we find that from 1996-2006, the Sun lost $\gtrsim 1.563 \times 10^{18} \mathrm{~g} \mathrm{yr}^{-1}$ via CMEs. This is a lower limit to $\dot{M}_{\mathrm{CME}}$ for the Sun because just under half of the reported CMEs from this time period have measured masses; there is also a bias against detection of the lowest mass and the widest CMEs. Despite the apparently relatively low association percentage of CMEs with flares $(\sim 13 \%$, A11), they contribute $40 \%$ of the total observed CME mass loss rate.

The total mass in our inferred solar CME mass distribution (filled histogram, Fig. 2) is $1.558 \times 10^{18} \mathrm{~g}$, equal to the observed LASCO CME mass loss rate within less than $1 \%$. Both the observed and inferred mass loss rates are $\sim 10^{-16} \mathrm{M}_{\odot} \mathrm{yr}^{-1}$, about two orders of magnitude less than the present-day solar wind $\left(10^{-14} \mathrm{M}_{\odot} \mathrm{yr}^{-1}\right.$; Li 1999).

This empirical method is particularly subject to observational biases on the low CME mass/low flare energy end of the distribution, one could fit a power law to the complete 
part of the distribution and analytically determine a mass loss rate as

$\dot{M}_{\mathrm{CME}}=\int_{M_{\min }}^{M_{\max }} \frac{\mathrm{d} N}{\mathrm{~d} M_{\mathrm{i}}} M_{\mathrm{i}} \mathrm{d} M$,

where $M_{\mathrm{i}}$ represents our inferred CME masses, and

$\frac{\mathrm{d} N}{\mathrm{~d} M_{\mathrm{i}}}=K_{\mathrm{M}, \mathrm{i}} M_{\mathrm{i}}^{-\gamma_{\mathrm{i}}}$.

We set $M_{\max }$ to the most massive CME observed, $\sim 6 \times 10^{16} \mathrm{~g} . M_{\min }$ is unknown due to observational bias, so we test a couple of observationally motivated cases. First, the completeness limit of the X-ray flare energy/frequency distribution: we translate this value into mass via Eq. (1), and find $M_{\min } \sim 10^{15} \mathrm{~g}$. The mass loss rate as calculated with Eq. (2) is then $6.6 \times 10^{-16} \mathrm{M}_{\odot} \mathrm{yr}^{-1}$. This represents a very conservative $M_{\min }$; there are clearly a number of CMEs less massive than $\sim 10^{15} \mathrm{~g}$. If we set $M_{\min }$ to the least massive solar CME observed, $\sim 10^{13} \mathrm{~g}$, the mass loss rate we infer is $2.2 \times 10^{-15} \mathrm{M}_{\odot} \mathrm{yr}^{-1}, \sim 10 \%$ of the presentday solar wind mass loss rate.

\subsection{T Tauri mass loss rates}

For the stellar case, we proceed as described for the solar case. The flare event rate/energy distribution measured by Albacete Colombo et al. (2007) for the COUP (Fig. 1, inset) and the CME mass/flare energy relationship (Eq. (1)) are combined to calculate an inferred stellar CME mass distribution (Fig. 3). Directly integrating this CME mass distribution (shaded histogram, Fig. 3), the inferred CME massloss rate is $6.2 \times 10^{-13} \mathrm{M}_{\odot} \mathrm{yr}^{-1}$.

In the stellar case we lack observational constraints on CME masses, but we can inform the mass loss calculations with X-ray observations. An equivalent way of expressing the mass loss rate in terms of the flare energy/frequency distribution, $\mathrm{dN} / \mathrm{dE}$, is

$\dot{M}_{\mathrm{CME}}=\int_{E_{\min }}^{E_{\max }} \frac{\mathrm{d} N}{\mathrm{~d} E} M_{\mathrm{i}} \mathrm{d} E$.

From Albacete Colombo et al. (2007), $\mathrm{d} N / \mathrm{d} E=K_{\mathrm{E}} E^{-\alpha}$, where $\alpha$ is 2.1 and $K_{\mathrm{E}}$ is $2.69 \times 10^{32}$ in cgs units. For $E_{\max }$, we simply adopt the maximum observed flare energy in the COUP sample, $\sim 10^{37} \mathrm{erg}$. For $E_{\min }$, we calculate what the minimum flare energy would need to be for the entire stellar X-ray luminosity (assuming a $1.0 \mathrm{M}_{\odot}$ star with $L_{\mathrm{X}} \sim$ $10^{30} \mathrm{erg} \mathrm{s}^{-1}$; Preibisch et al. 2005) to be produced solely by flares. Our analytically calculated stellar CME mass loss rate is then $\dot{M}_{\mathrm{CME}} \lesssim 3.2 \times 10^{-10} \mathrm{M}_{\odot} \mathrm{yr}^{-1}$. When the reported error on $\alpha$ is taken into account, the mass loss rate we estimate varies from $\sim 10^{-11}$ to $\sim 10^{-9} \mathrm{M}_{\odot} \mathrm{yr}^{-1}$. For comparison, mass loss rates in TTS winds have been reported to range from $\sim 10^{-12}$ to $\sim 10^{-7} \mathrm{M}_{\odot} \mathrm{yr}^{-1}$ (e.g., TW Hya and accreting stars in Taurus: Dupree et al. 2005; Muzerolle et al. 2000; Hartigan, Edwards \& Ghandour 1995).

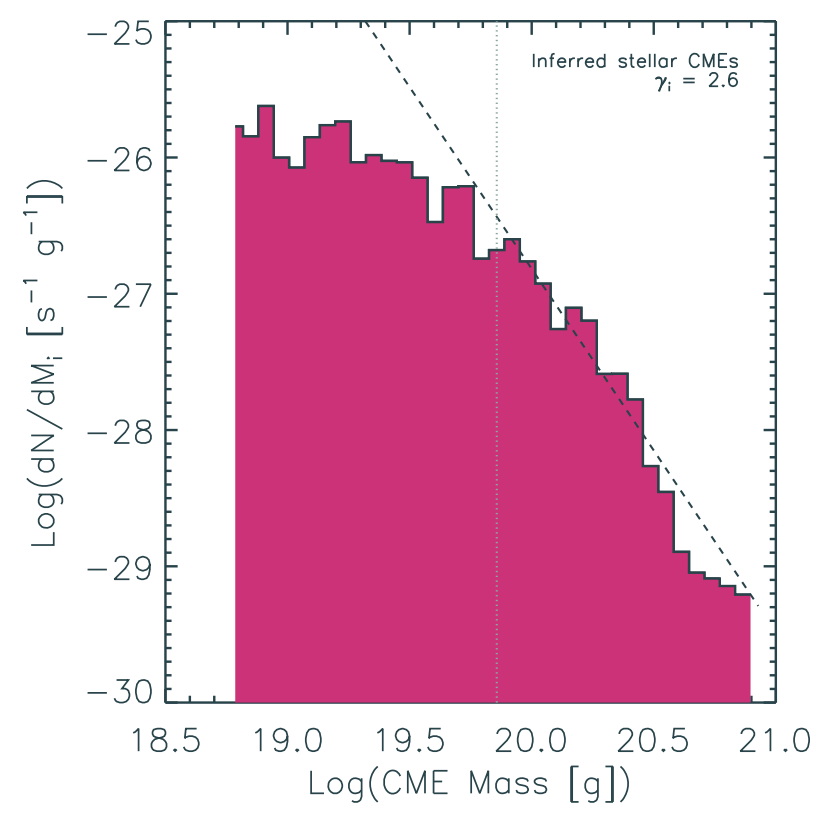

Fig. 3 (online colour at: www.an-journal.org) Inferred stellar CME distribution (filled histogram) with analytical fit (dashed line) to distribution above the flare energy/rate distribution of $\mathrm{Al}$ bacete Colombo et al. $\left(2007 ; 10^{35.6} \mathrm{erg}\right)$. The power law slope, $\gamma_{\mathrm{i}}$, is reported in the plot legend.

\section{Angular momentum evolution}

For the stellar CME case, we have inferred mass loss rates empirically and analytically, setting lower and upper limits of $10^{-12}$ and $10^{-9} \mathrm{M}_{\odot} \mathrm{yr}^{-1}$, respectively. To calculate the attendant torque against a protostar's rotation by mass loss at these rates, we need to estimate the lever arm length $\left(r_{\mathrm{A}}\right.$; e.g., Matt \& Pudritz 2008a and references therein). We treat the CME mass loss as a time-variable wind, per the prescription of Matt \& Pudritz (2008a) and Matt et al. (2012). Assuming the stellar field is globally dipolar with a strength of $600 \mathrm{G}$ (i.e., the equatorial dipole field strength of BP Tau, as reported by Donati et al. 2008) and an ideal MHD wind, the lever arm is

$\frac{r_{\mathrm{A}}}{R_{*}} \simeq 2.1\left(\eta \frac{B_{*}^{2} R_{*}^{2}}{\dot{M}_{\mathrm{CME}} v_{\mathrm{esc}}}\right)^{0.22}$.

The factor $\eta$, ranging from $0-1$, accounts for the wind's time-variability; folded into $\eta$ are terms describing the duration of each CME "burst," i.e., the time during which the CME mass loss is wind-like, and the fraction of the field which is open and participating in the mass loss. Note that $\eta=1$ is the case of a steady-state wind. The angle of the lever arm with respect to the stellar spin axis is also a factor in the torque, but the error introduced by $\eta$ is far greater, so we neglect the launch angle in this computation.

The torque, then, of the CME mass loss can be expressed as

$T_{\mathrm{CME}}=-\dot{M}_{\mathrm{CME}} \Omega_{*} r_{\mathrm{A}}^{2}$,

where $r_{\mathrm{A}}$ is as defined in Eq. (5). It is advantageous to express the torque's effect on the stellar rotation rate in terms 


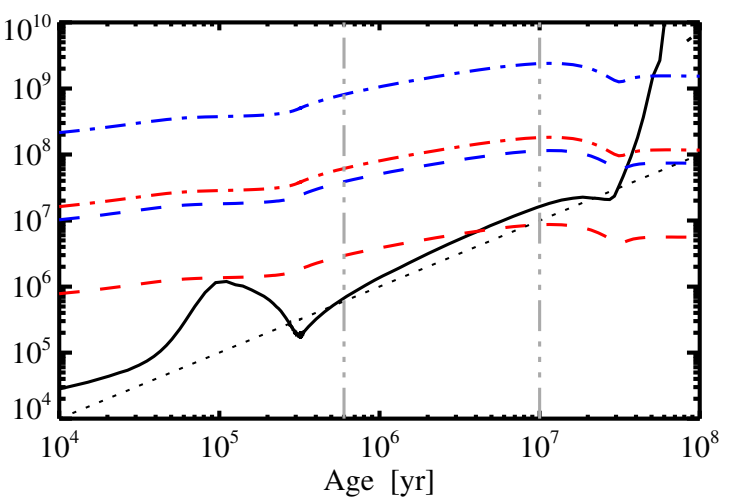

Fig. 4 (online colour at: www.an-journal.org) The spin-down timescale of varying stellar CME mass loss rates and spin-up due to contraction (solid, black line). The dotted black line indicates a constant rotation rate. Blue lines (upper dot-dashed and upper dashed) denote $\dot{M}_{\mathrm{CME}}=10^{-12} \mathrm{M}_{\odot} \mathrm{yr}^{-1}$, and red lines (lower dot-dashed and lower dashed) $\dot{M}_{\mathrm{CME}}=10^{-10} \mathrm{M}_{\odot} \mathrm{yr}^{-1}$. Dashdotted lines are the case of $\eta=10^{-3}$, while dashed lines are $\eta=$ 1. At any age, then, the lowest line on the plot is dominant in its effect on the stellar spin. Figure directly reproduced from Aarnio, Matt \& Stassun (2012b).

of spindown timescales, as it removes an initial rotation rate from the equation. We express the CME spindown timescale as

$\tau_{\mathrm{CME}}=\frac{I_{*} \Omega_{*}}{T_{\mathrm{CME}}}=k^{2}\left(\frac{M_{*}}{\dot{M}_{\mathrm{CME}}}\right)\left(\frac{R_{*}}{r_{\mathrm{A}}}\right)^{2}$,

where the moment of inertia of the star $\left(I_{*}\right)$ is calculated using a $1 \mathrm{M}_{\odot}$ Siess et al. (2000) model to describe the evolution of stellar parameters. We show the stellar spin up due to contraction and the CME spindown in Fig. 4. Clearly, contraction dominates stellar rotation for the first $\sim 30 \mathrm{Myr}$, but given a high enough CME mass loss rate $\left(10^{-10}-10^{-9}\right.$ $\mathrm{M}_{\odot} \mathrm{yr}^{-1}$ ) and favorable value of $\eta$, the CME torque could begin to dominate toward the end of the TTS phase.

\section{Discussion and conclusions}

In this work, we derive mass loss rates for pre-main sequence, solar type stars and find them to be relatively modest by comparison with mass loss rates observed for T Tauri winds. Our stellar CME model is derived from relating solar activity to stellar; in one sense, we immediately underestimate mass losses via CMEs by only selecting solar CMEs that occurred with flares. We find, however, that much of the mass loss comes from the CMEs associated with flares. The most massive CMEs are associated with the most powerful flares, and the most energetic flares are the most often associated with CMEs (A11). Thus, using stellar flare activity to determine a stellar CME rate likely represents a lower bound on the stellar CME activity.
This represents a first attempt at estimating mass and angular momentum losses via stellar coronal mass ejections. A more detailed treatment would consider additional external torques, i.e., it is likely that accretors could show an enhanced activity rate and thus more frequent CMEs, as well as accretion-driven winds Matt et al. (2008b) which would further deplete angular momentum. Additionally, time evolution of the stellar activity rate could be considered; here we assume it is constant. Our inferred CME mass loss rates range from $10^{-12}-10^{-9} \mathrm{M}_{\odot} \mathrm{yr}^{-1}$ for TTS, and we demonstrate how CMEs could substantially slow a solar mass star's rotation within the first 30 Myr. This relationship could potentially be adapted for stars of different masses, but careful consideration of the changing physical scales would be necessary (e.g., how the mass ejected scales with the total coronal mass, or how the lever arm/Alfvén radius scales with stellar radius).

Acknowledgements. A. N. A. acknowledges NSF grant AST0808072, P.I. K. G. S.

\section{References}

Aarnio, A. N., Stassun, K. G., \& Matt, S. P. 2010, ApJ, 717, 93

Aarnio, A. N., Stassun, K. G., Hughes, W. J., \& McGregor, S. L. 2011, Sol. Phys., 268, 195

Aarnio, A., Llama, J., Jardine, M., \& Gregory, S. G. 2012a, MNRAS, 421,1797

Aarnio, A. N., Matt, S.P., \& Stassun, K. G. 2012b, ApJ, 760, 9

Albacete Colombo, J.F., Caramazza, M., Flaccomio, E., et al. 2007, A\&A, 474, 495

Donati, J.-F., Jardine, M. M., Gregory, S. G., et al. 2008, MNRAS, 386,1234

Dupree, A. K., Brickhouse, N. S., Smith, G. H., \& Strader, J. 2005, ApJ, 625, L131

Favata, F., Flaccomio, E., Reale, F., et al. 2005, ApJS, 160, 469

Hartigan, P., Edwards, S., \& Ghandour, L. 1995, ApJ, 452, 736

Leitzinger, M., Odert, P., Ribas, I., et al. 2011, A\&A, 536, A62

Li, J. 1999, MNRAS, 302, 203

Massi, M., Ros, E., Menten, K. M., et al. 2008, A\&A, 480, 489

Matt, S. P., \& Pudritz, R. E. 2005, ApJ, 632, L135

Matt, S. P., \& Pudritz, R. 2008a, Understanding the Spins of Young Stars. in Cool Stars, Stellar Systems, and the Sun, ed. G. van Belle, ASPC 384 (ASP, San Francisco), 339

Matt, S., \& Pudritz, R. E. 2008b, ApJ, 678, 1109

Matt, S. P., Pinzón, G., de la Reza, R., \& Greene, T. P. 2010, ApJ, 714, 989

Matt, S. P., MacGregor, K. B., Pinsonneault, M. H., \& Greene, T. P. 2012, ApJ, 754, L26

Muzerolle, J., Calvet, N., Briceño, C., et al. 2000, ApJ, 535, L47

Orlando, S., Reale, F., Peres, G., \& Mignone, A. 2011, MNRAS, 415,3380

Preibisch, T., Kim, Y. C., Favata, F., et al. 2005, ApJS, 160, 401

Siess, L., Dufour, E., \& Forestini, M. 2000, A\&A, 358, 593

Skelly, M. B., Unruh, Y. C., Cameron, A. C., et al. 2008, MNRAS, 385, 708 